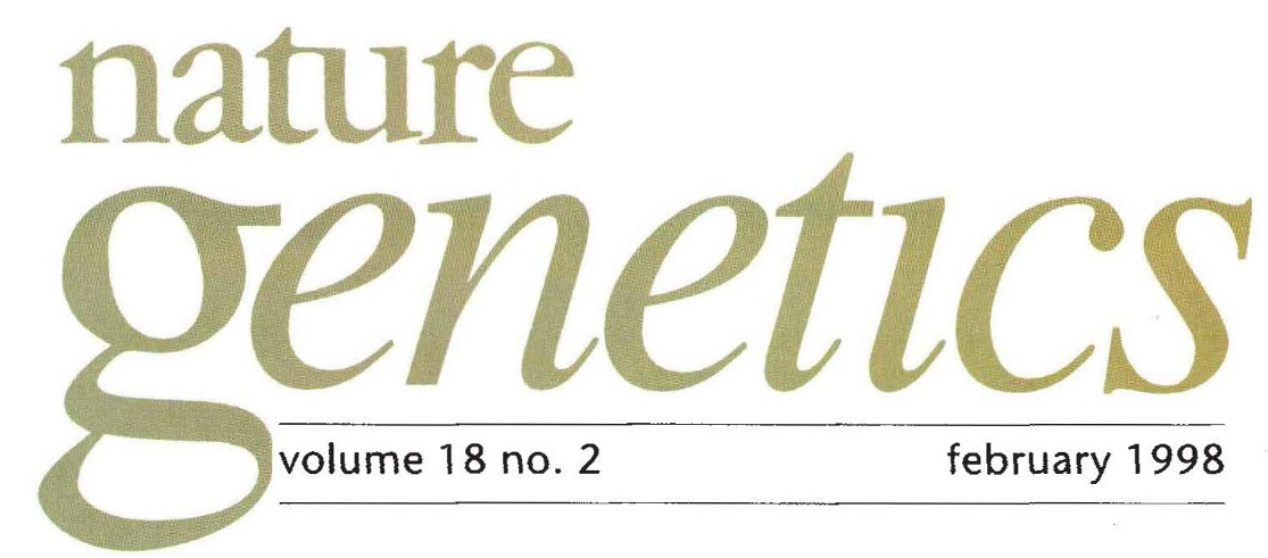

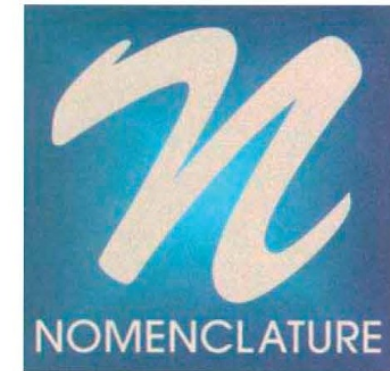

Belated New Year's resolution: bookmark this URL

http://genetics.nature.com/nomen/

\section{You say pto, I say Pto}

As symbols that represent and describe concepts and things, words are powerful. They facilitate, flavour and limit communication and the manner in which they are used has potent effect on the ear of the beholder. Their existence and evolution are dynamic, reflecting aspects of interaction between community and environment. So too, with gene symbols. It should therefore come as no surprise that there is much debate about nomenclature systems, and that those who co-ordinate them are working overtime to provide a coherent system in the face of the explosion of genetic information. The field of genetics is in the throes of a spectacular revolution and times of change are accompanied by changes in language; where development is concerned, the field of gene characterization is a neonate.

Both the traditional method of gene discovery - from mutant to gene-and 'reverse genetics' present particular challenges to a systematic means of establishing nomenclature. The former carries the weight of history; people become accustomed to using and become attached to gene names, and with good reason. The habit of language is peculiarly addictive - keen arguments are not uncommon and have even been known to result in "calling the whole thing off" over the mere pronunciation of the words: "tomato", "potato" and "neither". But once the function of a gene has been unequivocally established, a symbol that alludes to function is far more useful to the biologist than one that alludes to phenotype in the event of mutation. This is especially true in the long term, when additional homologous and associated genes will sift out of the various genomes - without systematic nomenclature, they could go unnoticed. The unknown aspect of the future, however, provides a major challenge to those who co-ordinate nomenclature: how to devise an adaptable, expandable system that can evolve as our knowledge of genes evolves. Clues can be obtained from other organisms - for example, the novel mouse ubiquitin E3 ligase reported on page 143 of this issue has an 'interim' name (for the short term, it is to be called Itch, a name derived from the mutant phenotype) until its molecular relationship with other known ligases can be determined. For this reason, and with the interests of the burdgeoning numbers of geneticists who seek to explore gene function through model organisms in mind, extensive efforts are now being made to standardize nomenclature across species and also, to create a database through which orthologues and orthologous families can be identified, despite having different nomenclature in some cases. As Lois Maltais, who co-ordinates the mouse nomenclature database puts it: "the emphasis is no longer on the gene as a single entity, but how groups of genes work together." 
The conversion from 'phenotypic' to 'functional' nomenclature can be painful, as witnessed by the understandable resistance of Drosophila geneticists to relinquish their delicious gene names - a taste of things to come for those with an eye for zebrafish nomenclature. The argument, however, that phylogenetic relationships can be determined using databases, and that there is no need for 'live' conversion in spoken language, holds little water; the spoken word informs perception. This tension can be attenuated by recognition of the fact that people are capable of speaking more than one language at a time, that those, say, in the Drosophila community may be most comfortable with the traditional, 'phenotypic' names, but as the advantages of a systematic nomenclature become more obvious, will become fluent in it as well.

Naming the increasing number of genes that bear no connection with phenotype, genes whose identities are simply realized by sequence motifs (if one is lucky), illustrates another challenge. The evolutionary effect of chopping and changing sequence motifs means that there are a plethora of genes that could be as well placed in one family as a completely different one. Even after gene function has been established in the laboratory, additional experiments may reveal an entirely different function, again indicating that its place in another gene family could be equally or more appropriate. Nomenclature curators, like editors, co-ordinate information and thus depend upon and actively welcome contact with geneticists who are able to advise on gene function and emerging relationships between genes. The human and mouse nomenclature committees actively encourage investigators in specific fields to set up their own committees - a strategy which allows people in these fields to propose schemes that are optimally designed in terms of scientific content and with respect to likely acceptance by the community. As Julia White, of the HUGO nomenclature committee points out, "there is no point in having a wonderfully elegant system that beautifully reflects phylogenetic relationships, if no one remembers what the symbols are". More specialist advisors are sought: for example, those with expert knowledge of genes associated with the apoptosis-signalling cascade (which includes genes with such unrelated symbols as DAXX, RIP, CLARP, TRADD and $P D C D 1$ ) are especially welcome at the present time.

In recognition of the need for improved dialogue between the scientific community and nomenclature curators, and also, for a common genetic language, Nature Genetics now requests that authors consult with the appropriate nomenclature committee prior to reporting the discovery of a novel gene. In cases where a symbol cannot be decided upon before publication, a provisional or temporary symbol can be used. Establishing an appropriate, approved symbol not only contributes to the smoother evolution of a common language; it also increases the odds that this symbol will become permanent. Similarly, use of approved gene symbols, where they exist, will also be required. The use of symbols such as TP53 (instead of p53) and CDKNIA (instead of p21) may irritate at first sight, but active and regular use quickly etches the neural grooves and biases toward even more regular use and acceptance. A brief guide to nomenclature protocol and an article that reviews the whys and wherefores of various nomenclature systems in different organismsreplete with live links to the appropriate databases - can now be found on the Nature Genetics website (http://genetics.nature.com/nomen/). Its authors-Julia White, Lois Maltais and Dan Nebert (the latter of the University of Cincinatti) rightly point out that with the various genome projects in full-swing and the rate of gene discovery entering log phase, additional funding for nomenclature resources is desperately required. As Dan Nebert puts it, the "view that nomenclature is like some sort of hobby for some of us who are [perceived to be] obsessive and like to organize everything to do with nomenclature in our evenings and week-ends" is shortsighted and misinformed. The nomenclature committees deserve support from the scientific community, in practice and materially. 